\title{
Modal Analysis of a Small Smooth Kart Tire - Numerical and Experimental Determination
}

\author{
Chukwuemeke William Isaac \\ University of Ibadan, Mechanical Engineering Department, Ibadan, Nigeria.
}

\begin{abstract}
(Received 15 July 2016; accepted 31 January 2017)
The modal analysis of a kart tire was performed using the Abaqus/explicit finite element numerical software and the results obtained were validated by experimental testing. Frequency response functions, damping ratios, and modal shapes are the driving parameters for analysis. The investigation shows a very useful modal response of the kart tire such that a high frequency response function of approximately $301 \mathrm{~Hz}$ to $630 \mathrm{~Hz}$ was obtained for both the finite element solutions and experimental results. These results are much higher than that of the conventional tires therefore making it an excellent tire-type material that can be used for further analysis and study of tire-road contact problems, air-pumping, and noise radiation.
\end{abstract}

\section{INTRODUCTION}

A major source of noise generated from tire-road interaction has been attributed to vibration of air molecules inside the tire cavity. The vibration and dynamic response of tires have been investigated by a number of researchers by adopting both numerical and experiment methods. A numerical approach such as the finite element method (FEM) has proved to be an efficient modelling tool that is employed by researchers to overcome some of the inherent difficulties such as the complexity in the tire geometry and the material properties for which the modelling of tires by analytical methods remain intractable. Different authors have carried out finite element (FE) simulations and analyses to predict the dynamic response of tires. ${ }^{1,2}$ Also, the finite element approach was performed by Chatterjee and Ranjan to analyse the free vibration of radial pneumatic tires. ${ }^{3}$ The authors obtained the natural frequencies and mode shapes of tires. They also investigated the effects of some parameters such as the inflation pressure and tread patterns on the natural frequency of tires. In their findings, it was observed that an increase in inflation pressure resulted in an increase in both stiffness of the tire and its natural frequencies at some optimum values. Also, for lower modes, the natural frequencies did not vary significantly with smooth tires or patterned tires, but with higher modes, the natural frequencies for patterned tire increased significantly more than smooth tires. They concluded that the increase in the ply-angle and variation of belt thickness had no significant effect on the natural frequencies of the tires. The dynamics of rolling tires can also be described by an Arbitrary Lagrangian Eulerian (ALE) formulation. Brinkmeier et al. investigated the analysis of tire-road noise using the FEM. ${ }^{4}$ In their investigation, the non-linear stationary rolling case was described by an ALE formulation. The deformation of the motion into rigid body motion was described in an Eulerian manner whereas the large deformation was measured with respect to the intermediate reference in Lagrangian coordinates. The vibration of a tire model can also be analysed by the wave finite element method (WFEM). This approach has the advantage of modelling vibration at higher frequencies for which the classical FE model becomes impracticably large. Yoshiyuki et al. applied this method to model the vibration of a tire using ANSYS by taking the material properties of the rubber as frequency dependent and the wave propagation characteristics were extracted from the dynamics stiffness matrix of the FE model. ${ }^{5}$ They determined the free and forced vibration of the tire and concluded that the WFEM is a powerful tool used for predicting the dynamic behaviour of a complex structure.

Modal analysis has been used to estimate tire parameters especially in the orthotropic plate model of the tire. Périsse and Hamet analytically modelled a radial tire vibration by comparing a $2 \mathrm{D}$ ring and $3 \mathrm{D}$ orthotropic plate for low and high frequency ranges. ${ }^{6}$ They derived the natural frequencies and mode shapes from the ring and plate theory. In their findings, the $2 \mathrm{D}$ ring model was valid at low and medium frequency range, i.e., $400 \mathrm{~Hz}$, while the $3 \mathrm{D}$ plate model was valid over the whole frequency range, i.e., 0 to $2000 \mathrm{~Hz}$ and at higher frequencies. When the wavelength $\lambda$ approximates the tread width $l$ of the tire, i.e., $\lambda \approx l$, the tire becomes a two dimensional waveguide and the plate model has to be used. Modelling of tire-road interaction can be determined by evaluating the time response, also known as the Green's Function of the tire, to a concentrated external force. The response of a tire to a contact force is mostly determined in the time domain because of the non-linearity of the contact problem. Lopez et al. determined the response of a rotating tire in a fixed reference frame using the Green's Function. ${ }^{7}$ The authors found out that the Green's Function for a rotational velocity 0 to $100 \mathrm{rad} / \mathrm{s}$ differs considerably both in frequency and amplitude. The frequency shift was correctly captured in the derived Green's Function while the amplitude decreases due to rotational velocity caused by the increase of damping due to rotation. The effect of rotation on the dynamic behaviour of a rolling tire is also very crucial when modelling tire-road noise caused by vibration. Lopez et al. modelled vibrations on a deformed rolling tire at low frequencies and determined the eigenvalues and eigenmodes. ${ }^{8}$ The response of the rotating tire in a fixed reference frame and the gyroscopic and centrifugal effect were calculated. The authors also examined the deformation on the eigenfrequencies of a rotating tire. ${ }^{9}$ Their results showed that the eigenfrequencies of a deformed tire decreases rapidly with increase in velocity while for an undeformed tire, no modal reaction due to rotation was observed. Modal interactions increase as the load on the tires increases and decrease as the material damping increases. The characteristics of a rotating tire can also be predicted by determining the natural frequencies and their wave-like basis functions. Kim and Bolton modelled the treadband of an inflated, rotating circular cylindrical shell 
Table 1. Material properties of kart tire parts.

\begin{tabular}{||c|c|c|c|c||}
\hline \hline Tire part & $E(\mathrm{GPa})$ & $\rho\left(\mathrm{kg} / \mathrm{m}^{3}\right)$ & $\nu$ & $t(\mathrm{~mm})$ \\
\hline Tread & 0.085 & 1150 & 0.49 & 10.0 \\
\hline Carcass & 0.81 & 3900 & 0.44 & 4.8 \\
\hline Steel beads & 27.32 & 5900 & 0.33 & 3.8 \\
\hline
\end{tabular}

to identify the effects of rotation. ${ }^{10}$ They used a selection procedure to associate each wave-like basis function with a single natural frequency and obtained analytical solutions of both free and forced vibration cases. Their results showed two effects: stiffening of the treadband and kinematic "tilting" of the dispersion curves. These dispersion curves could be used to analyse the potential of a rotating tire to radiate sound. The noise generated by tire vibration of vehicles represents the largest component of the total noise generated by vehicles. In both urban and rural areas, it could be a source of disturbance.

In recent years, attempts have been made to validate the numerical results of the modal analysis of standard tires. ${ }^{11-13}$ In spite of the numerical and experimental approaches to reduce the effect of tire road noise, it is observed that the natural frequencies of vibrating tires at different modes are still within the range of 100 to $300 \mathrm{~Hz}$. These low frequency ranges are insufficient to effectively characterize tire vibration, air pumping, or noise radiation. Higher natural frequencies are required initially to fully characterize tire behaviour during vibration and tires rolling with or without contact to the ground. This study aims to obtain the fundamental modal parameters of tires such as the natural frequencies, damping ratios, and modal shapes. It adopts a very unique type of tire which has less complicated tire structure and the natural frequencies obtained are comparatively high enough to characterize tire response during vibration and air pumping.

\section{MATERIALS AND METHODS}

\subsection{Problem Description}

The tire parts-tread, carcass, and steel beads as shown in Fig. 1(a) — are modelled by a three — dimensional deformable shape. The analysis is performed on a smooth kart tire, size 100/55R1.8. The tire has a smooth full tread without pattern or grooves. An air cavity inside the tire is modelled with varying distributed pressures ranging from 100 to $200 \mathrm{kPa}$. The finite element software used is Abaqus 6.12.1. Validation of the model was effected by comparing the measured natural frequencies to the natural frequency extraction computed in Abaqus. For the boundary condition, the centre of the wheel is free to rotate along its axis and all the other directions are constrained. A steady state dynamic was used to obtain the Frequency Response Function (FRF). An excitation force in the radial direction was applied at the centre of the tread-band. A frequency resolution for the simulation ranges from 80 to $1000 \mathrm{~Hz}$. The tread is made of rubber $10 \mathrm{~mm}$ thick and tread-band of $80 \mathrm{~mm}$ thick which was modelled as an incompressible elastic material. The carcass was modelled as two elastic layers of rubber-coated nylon cord $4.8 \mathrm{~mm}$ thick while the twelve beads were modelled as an elastic onedimensional beam element $3.8 \mathrm{~mm}$ thick.

\subsection{Material Properties}

The material properties of tread, carcass, and steel beads are seen in Table 1. A linear isotropic elastic material model is adopted given as:

$$
\begin{aligned}
& \left\{\begin{array}{c}
\epsilon_{11} \\
\epsilon_{22} \\
\epsilon_{33} \\
\gamma_{12} \\
\gamma_{13} \\
\gamma_{23}
\end{array}\right\}=\left[\begin{array}{cccccc}
1 / E & \nu / E & -\nu / E & 0 & 0 & 0 \\
-\nu / E & 1 / E & -\nu / E & 0 & 0 & 0 \\
-\nu / E & -\nu / E & 1 / E & 0 & 0 & 0 \\
0 & 0 & 0 & 1 / G & 0 & 0 \\
0 & 0 & 0 & 0 & 1 / G & 0 \\
0 & 0 & 0 & 0 & 0 & 1 / G
\end{array}\right] \\
& \times\left\{\begin{array}{l}
\sigma_{11} \\
\sigma_{22} \\
\sigma_{33} \\
\sigma_{12} \\
\sigma_{13} \\
\sigma_{23}
\end{array}\right\} .
\end{aligned}
$$

Where $\epsilon, \gamma$ and $\sigma$ are the strains and stresses, respectively, while $E$ is the Young's modulus and $\nu$ is the Poisson ratio. The shear modulus is given by:

$$
G=\frac{E}{2(1+\nu)} .
$$

The frequency domain or time domain can be adopted to accurately identify the response of the viscoelastic material of the kart tire. For the simulation, the time domain was adopted to identify the viscoelastic material response. For small strain, a viscoelastic material model for the response which is the shear stress can take the form:

$$
\tau(t)=\int_{0}^{t} G_{r}(t-s) \dot{\gamma}(s) d s
$$

Where $G_{r}$ is the shear modulus at small strain. For a zero time $(t)$ at the beginning of the simulation, the shear relaxation modulus can be written as:

$$
g_{r}(t)=\frac{G_{r}(t)}{G_{0}}
$$

Where $G_{0}$ is the instantaneous shear modulus. The expression for the shear stress takes the form:

$$
\tau(t)=G_{0} \int_{0}^{t} g_{r}(t-s) \dot{\gamma}(s) d s .
$$

In the time domain, it is defined by the Prony series expansion of the dimensionless relaxation 14 expressed as:

$$
g_{r}(t)=1-\sum_{i=1}^{N} \bar{g}_{i}^{P}\left(1-e^{-t / \tau_{i}^{G}}\right)
$$

Where $N, \bar{g}_{i}^{P}$ and $\bar{\tau}_{i}^{G}$ for $i=1,2, \ldots N$ are the material constants.

For linear isotropic elasticity, the shear stress is given as:

$$
\tau(t)=G_{0}\left(\gamma-\sum_{i=1}^{N} \frac{\bar{g}_{i}^{P}}{\tau_{i}^{G}} \int_{0}^{t} e^{-s / \tau_{i}^{G}} \gamma(t-s) d s\right) .
$$

For both linear anisotropic elasticity and finite strain, the Prony series expansion for the deviatoric stress are respectively given as:

$$
\tau=\tau_{0}-\sum_{i=1}^{N} \frac{\bar{g}_{i}^{P}}{\tau_{i}^{G}} \int_{0}^{t} e^{-s / \tau_{i}^{G}} \tau_{0}(t-s) d s
$$


Table 2. Viscoelastic properties of tread component. ${ }^{15}$

\begin{tabular}{|c|c|c|c|c|}
\hline \multirow{2}{*}{ Material } & \multicolumn{2}{|c|}{ Shear relaxation modulus } & \multicolumn{2}{c|}{ Relaxation time } \\
\cline { 2 - 5 } & $g_{1}$ & $g_{1}$ & $\tau_{1}$ & $\tau_{2}$ \\
\hline Tread & 0.1433 & 0.0852 & 7.6125 & 235.62 \\
\hline
\end{tabular}

$$
\begin{aligned}
& \tau=\tau_{0}- \\
& \sum_{i=1}^{N} \operatorname{dev}\left(\frac{\bar{g}_{i}^{P}}{\tau_{i}^{G}} \int_{0}^{t} e^{-s / \tau_{i}^{G}} \bar{\nabla}_{t}^{-1}(t-s) .\right. \\
& \left.\tau_{0}(t-s) \bar{\nabla}_{t}(t-s) d s\right) .
\end{aligned}
$$

Where $\tau_{0}$ is the instantaneous deviatoric stress, $\bar{\nabla}_{t}$ is the deformation gradient at time $(t-s)$.

To model the tread component of the kart tire, double values were defined directly for the shear relaxation modulus ratio $\bar{g}_{i}^{P}$ and its associated relaxation time $\tau_{i}$ in the Prony series expansion. These values were obtained from Yang ${ }^{15}$ as shown in Table 2.

In the model, to take into account the non-linear and incompressible material behaviour of the rubber which is typically the tread component of the kart tire, the strain energy for the Mooney Rivlin formation was adopted and the stress ratios $l a m b d a_{i}$ are used to describe the stresses defined as:

$$
\begin{gathered}
\bar{I}_{1}=\sum_{i=1}^{3} \lambda_{i}^{2} ; \\
\bar{I}_{2}=\sum_{i=1}^{3} \lambda_{i}^{-2} .
\end{gathered}
$$

Where $\bar{I}_{1}$ and $\bar{I}_{2}$ are the first and second deviatoric strain invariants. The principal stresses $\lambda_{i}$ are expressed in terms of the principal strains $\epsilon_{i}$ given as:

$$
\lambda_{i}=1+\epsilon_{i} .
$$

The strain energy $U$ for the Mooney Rivlin formulation is generally expressed as:

$$
U=C_{10}\left(\bar{I}_{1}-3\right)+C_{01}\left(\bar{I}_{1}-3\right)+\frac{1}{D_{1}}\left(J^{*}-1\right)^{2} .
$$

Where $C_{10}, C_{01}$ and $D_{1}$ are material parameters which are temperature dependent. $J^{*}$ is the elastic volume ratio. However, in this study, the material behaviour is independent of the temperature and Eq. 13 is simplified to:

$$
U=C_{10}\left(\bar{I}_{1}-3\right)+C_{01}\left(\bar{I}_{1}-3\right) .
$$

The coefficients $C_{10}$ and $C_{01}$ are respectively 0.51 and 1.86 and are obtained from Chae. ${ }^{16}$

\section{MODAL ANALYSIS AND RESPONSE}

\subsection{Modal Parameters and Eigenvalue Extractions}

Modal analysis was performed to identify the modal properties of the frequency modes. The modal parameters consist of the frequency, damping, and mode shapes for each eigenmode. A frequency extraction step was first performed to extract the natural frequencies. Lanczos eigen solver from Abaqus 6.12 documentation was adopted for the model and forty eigenvalues were extracted. During the eigenvalue extraction, the damping $[C]$ was neglected and the stiffness $[K]$ was assumed to be positive semi-denite for a symmetric eigen problem. The problem can be written as:

$$
\left(\omega^{2}[M]+[K]\right)\{\phi\}=0 .
$$

Where $\omega$ is the circular frequency. A set of simulations are performed given the spectral transformation as:

$$
[M]([K]-\psi[M])^{-1}[M]\{\phi\}=\theta[M]\{\phi\} .
$$

Where $\phi$ is the shift, $\theta$ are the eigenvalues and $\Phi$ are the eigenvectors. One can relate the eigenvectors and eigenvalues of Eqs. 15 and 16 as:

$$
\omega^{2}=\frac{1}{\theta}+\phi
$$

An advantage of using the Lanczos eigensolver is the possibility of computing many eigenmodes and rapid convergence to the desired eigenvalues. In this simulation, Rayleigh damping was used and it should be noted that eigenvectors of both damped and undamped systems are the same when Rayleigh damping is used.

\subsection{Frequency Response Function}

Frequency Response Function (FRF) is defined as the ratio of the response to the excitation force. There are three bases of FRFs according to the type of response parameters. They include displacement, velocity, and acceleration response functions. When the response parameter is displacement, a receptance FRF is obtained. If the response parameter is velocity, it is called mobility FRF, and if the response is acceleration, it is called inertance or accelerance FRF. For our simulation, a harmonic force was applied to excite the tire which produced an acceleration response thereby enabling us to find the inertance FRF $H(\omega)$. The equation of motion is given by:

$$
m \ddot{u}+c \dot{u}+k u=f(t) .
$$

Where $m, c$, and $k$ are the mass, damping, and stiffness, respectively. For the harmonic force applied to excite the tire, we have:

$$
f(t)=F e^{i \omega t} .
$$

Where the displacement $u$, velocity $\dot{u}$ and acceleration $\ddot{u}$ are respectively given as:

$$
\begin{gathered}
u(t)=U e^{i \omega t} ; \\
\dot{u}=i \omega U e^{i \omega t} ; \\
\ddot{u}=-\omega^{2} U e^{i \omega t} .
\end{gathered}
$$

Then the inertance FRF can simply be written as:

$$
H(\omega)=-\omega^{2} H_{d}(\omega) .
$$

Where $H_{d}$ is the receptance FRF.

\subsection{Determination of the Damping Ratio from the FRF Plot}

The damping ratios of the modal analysis were determined by applying the half power point method as described by Bilošová. ${ }^{17}$ These are points on the plot of magnitude of $H_{A}(\omega)$ in which the magnitude decreases to the value $H_{A} \sqrt{2}$ which means to the $1 / \sqrt{2}$ of the peak value. Let $H_{A}$ be the peak magnitude and $H_{P}$ be the half power value. Plotting the 


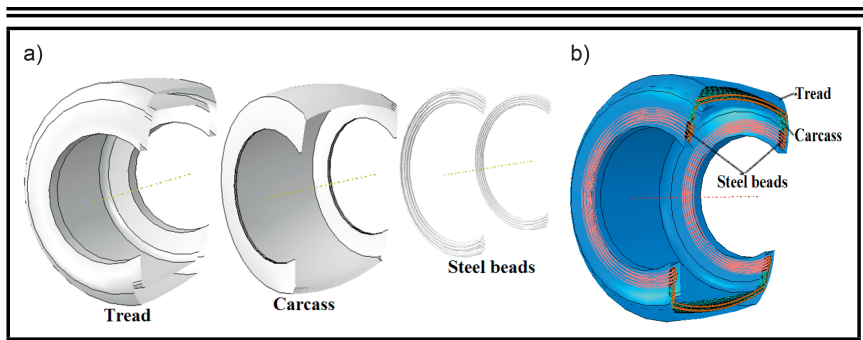

Figure 1. Geometrical configuration of element parts: (a) exploded section and (b) assembled section.

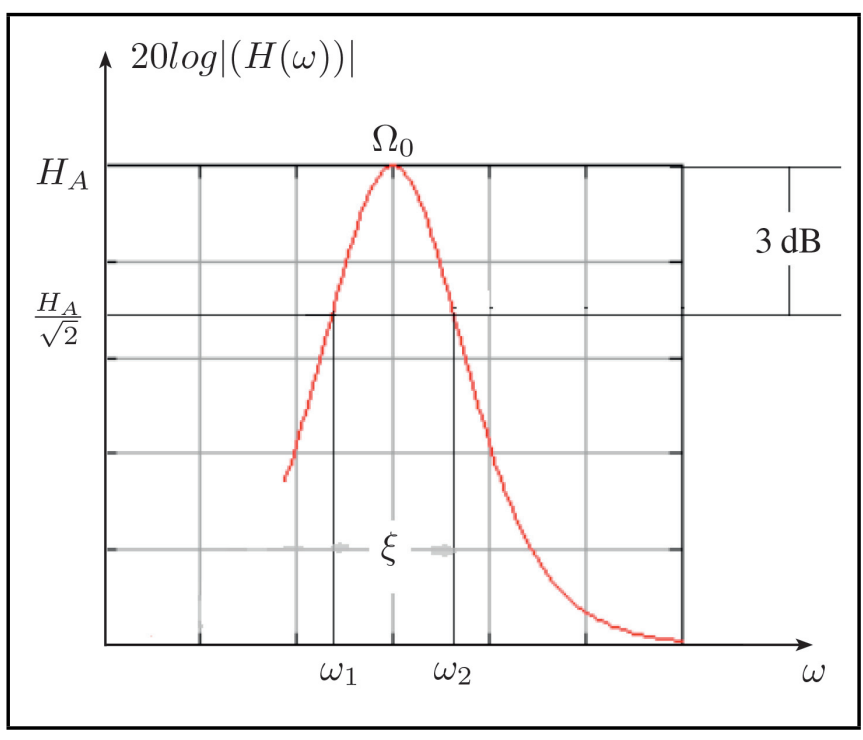

Figure 2. Determination of damping from the FRF logarithmic scale.

Table 3. Viscoelastic properties of tread component. ${ }^{15}$

\begin{tabular}{|c|c|}
\hline Tire part & Number of elements generated \\
\hline Tread & 84528 \\
\hline Carcass & 37441 \\
\hline Beads & 728392 \\
\hline
\end{tabular}

FRF in the logarithmic scale, the peak magnitude decreased by $3 \mathrm{~dB}$ as shown in Fig. 2. The difference between the peak value and the half power point value is $3 \mathrm{~dB}$. If the peak frequency is $\Omega_{0}$ and the damping ratio is $\xi$ then, the frequency at the root mean square value of $\omega_{1}$ and $\omega_{2}$ is given as:

$$
\omega_{2}-\omega_{1}=2 \xi \Omega_{0}
$$

\section{FINITE ELEMENT METHOD}

\subsection{Mesh Generation}

By finite element (FE), we can divide the tire structure into small, discrete sub-regions called elements which are joined at specific points called nodes located on their boundaries to form a mesh. Forces are transmitted by the nodes from one element to the other. These elements could be one, two-, or three- dimensional elements and the model may consist of a few axisymmetric elements or many thousands of solid (i.e., tread), membrane (i.e., carcass) or beam (i.e., steel beads) elements as represented in Fig. 1(a). The assembled finite element tire section is shown in Fig. 3. For the analysis, the number of elements generated is shown in Table 3.

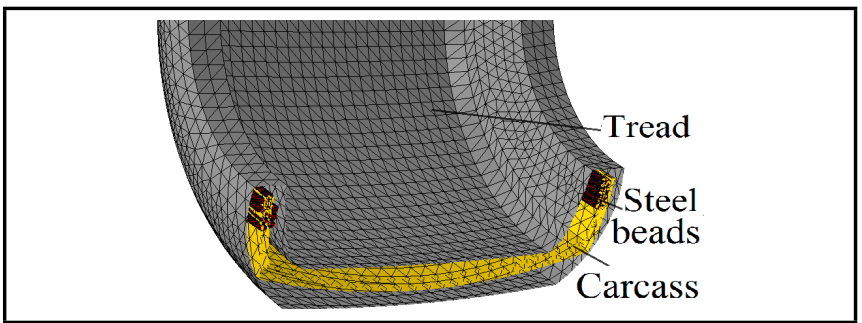

Figure 3. Assembled FE mesh of the kart tire section.

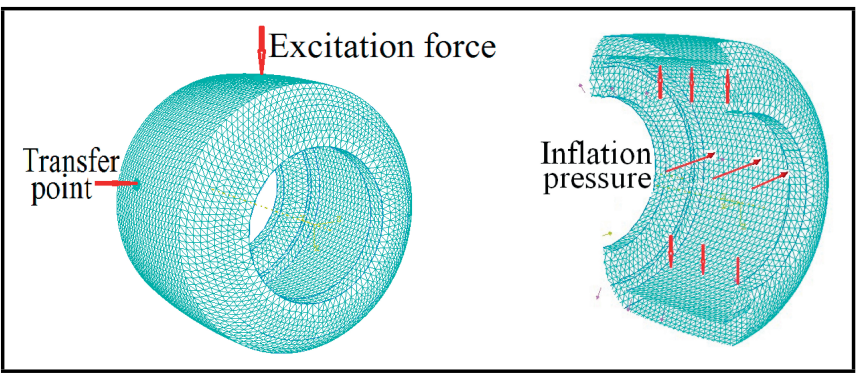

Figure 4. Location of nodes and boundary condition for FRF evaluation.

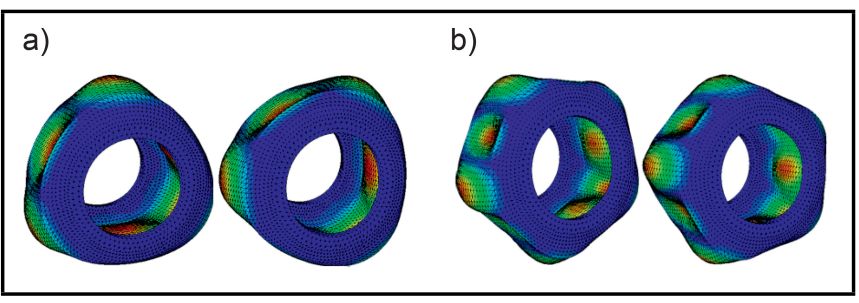

Figure 5. Pair of mode shapes: (a) $361.08 \mathrm{~Hz}$ and $361.74 \mathrm{~Hz}$; (b) $517.97 \mathrm{~Hz}$ and $518.66 \mathrm{~Hz}$

\subsection{Load and Boundary Conditions}

To ensure a modal dynamic response, a concentrated force $(F)$ of $1 \mathrm{~N}$ was applied at the centre of the tire in the radial direction. A transfer node away from the excitation point was considered to evaluate the transfer function. The transfer point was selected slightly away from the initial excitation point and a force of $1 \mathrm{~N}$ was applied at this point as shown in Fig. 4. In both cases, inflation pressure $P_{n}$ between 100 to $200 \mathrm{kPa}$ was applied as depicted in Fig. 4. Boundary conditions were given to the tire at the rim side wall such that the motion $\mu(x, y, t)$ on the rim is zero at $y=0, y=b_{y}$. The vibrational magnitude in the axial $\mathrm{z}$-direction and the corresponding eigenmode at the peak of resonance can be observed.

\section{NUMERICAL RESULTS AND VALIDATION}

\subsection{Mode shape}

The elastic material properties of the rubber were obtained from the work of Chatterjee and Ranjan ${ }^{3}$ but with a slight modication in the Young's modulus of the tread layer as obtained from Andersson and Larsson. ${ }^{18}$ For the tire simulation, a pair of modes appears at two different frequencies as illustrated in Fig. 5 with the mode shapes having real or standing waves.

These mode shapes agree with the mode shapes of the tire FR 70-14 obtained analytically by Soedel and Prasad ${ }^{19}$ when it is not in ground contact, and the smooth tire obtained by Périsse et al. who observed six modal shapes. ${ }^{20}$ Also, Meftah showed analytical results of three inextensible modal deformations of a circular ring ${ }^{21}$ while Tong and Jim obtained five 


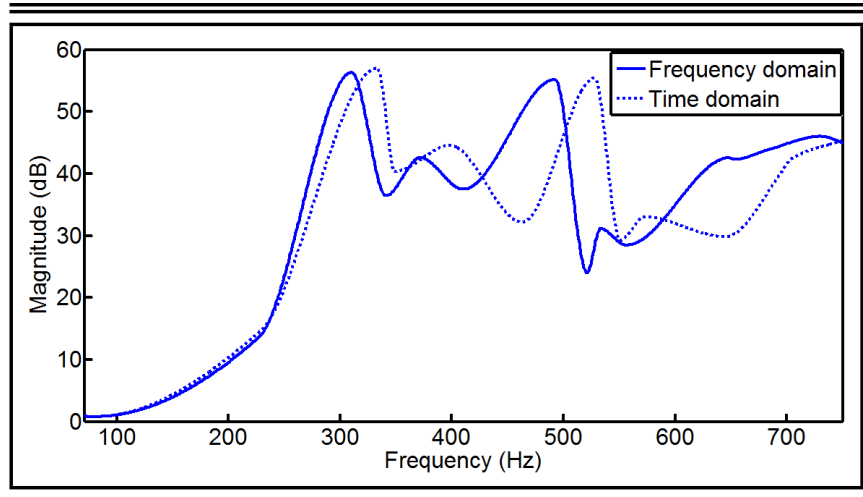

Figure 6. FRF comparison between frequency and time domain at excitation point.

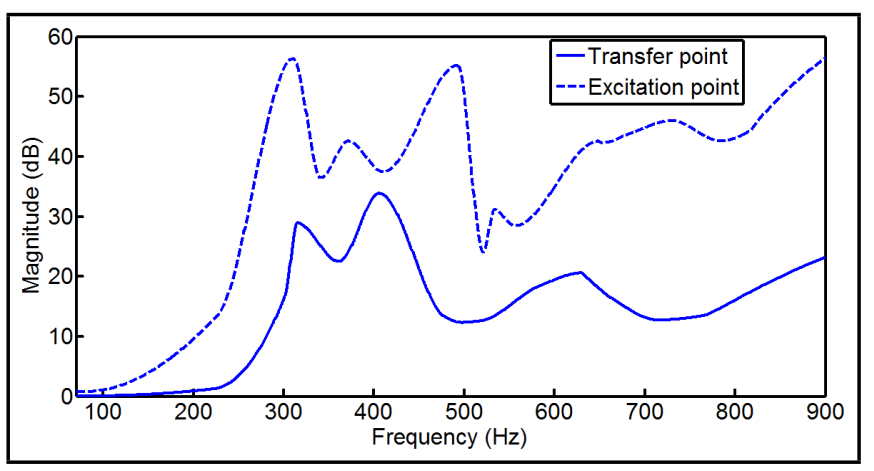

Figure 7. FRF comparison between excitation and transfer points in the frequency domain.

modal shapes of the Goodyear 195/65R15 tire t2 $^{22}$ which conforms with the mode shapes obtained by the FE simulation of kart tire. It is worth noting that with the kart tire simulation, a considerable higher frequency range can be obtained.

\subsection{Frequency Response Function Analysis}

The general Frequency Response Functions of the tire is obtained for the elastic material at excitation point both in the frequency and time domain as represented in Fig. 6. The peak width of these FRF determines the degree of damping and the peak heights gives the amplitude of vibration. At the excitation point, the first peak occurs in the range of 300 and $310 \mathrm{~Hz}$. The sharpness of the peak obtained at each natural frequency decreases with frequency. As more natural frequencies progressively occur, the peak width for each mode becomes less sharp, consequently resulting in a highly damped system. At higher frequencies, i.e., $800 \mathrm{~Hz}$, damping is difficult to determine.

At the transfer points, not all the modes are observed, and only four modes are visible. This may be due to zero resonance which occurs at the points of zero magnitude for the second, fourth, and seventh mode as show in Fig. 7. One observes that the amplitude of vibration decreased as vibration moves away from the excitation point. The amplitude at the transfer point is reduced by approximately $50 \%$ as compared to the amplitudes at the excitation point.

\subsection{Effect of Inflation Pressure}

The results of four different inflation pressures from 100 to $200 \mathrm{kPa}$ are shown in Fig. 8 at excitation and transfer points. Increase in natural frequencies, which are higher than the conventional tires, are obtained. It is also observed that the inflation pressure slightly affects the natural frequency due to the a)

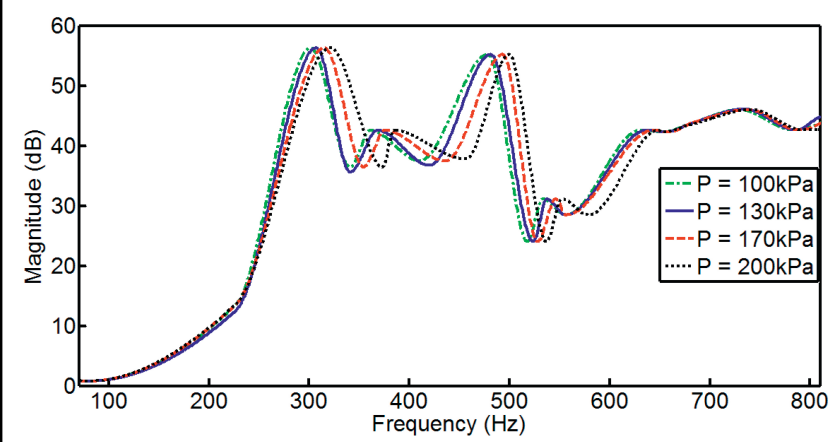

b)

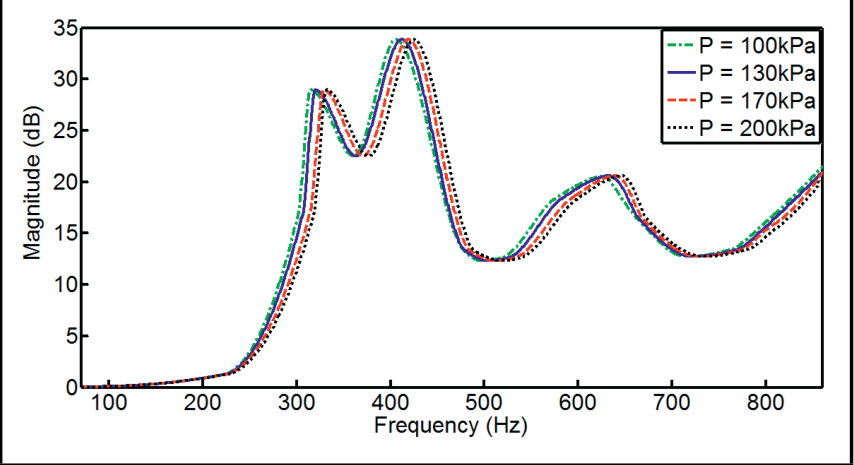

Figure 8. Influence of various inflation pressure on the natural frequency at: (a) excitation point and (b) transfer point.

stiffness of the tire. However, there is no significant change in the mode shape and no significant increase in the magnitude.

\subsection{Damping Ratio Evaluation}

The damping ratio $\xi$ was obtained for each natural mode by applying the methods explained in Section 3.3 and the procedures highlighted by Cosson and Rozycki. ${ }^{23}$ Investigation shows that at higher frequencies, the damping ratio was difficult to obtain for the tire simulation due to its highly damped property. Therefore, only the rst ve damping ratios were obtained. The modal damping based on measurement was obtained from the half-power bandwidth evaluation of the first five resonance peaks. Figure 9 shows the modal damping history plots of the frequency before resonance (FBR) against the frequency after resonance (FAR). The loss factor ranges from 0.075 at low frequencies to a maximum of 0.13 at high frequencies.

\subsection{FE Modal Response Validation}

To validate the FE simulation results, modal analysis was investigated experimentally to obtain the natural frequencies, damping ratios, and mode shapes. A Single Input Multiple Output (SIMO) as explained by Ewins ${ }^{24}$ was used to obtain the FRF at several measured points but on the same singlepoint excitation. Measurement was carried out on the tire by first measuring some points and then freely suspending the tire at the rim. Figure 10(a) shows a schematic representation of the experimental set up of the tire. The tire is excited radially using an electrodynamic shaker which is fed with a swept sine signal. The impedance head is a force transducer which has the force and acceleration sensors mounted on it and it is screwed to an aluminium plate of dimension $1 \times 1 \mathrm{~cm}$ which is then glued onto the surface of the tire. The stinger is placed 


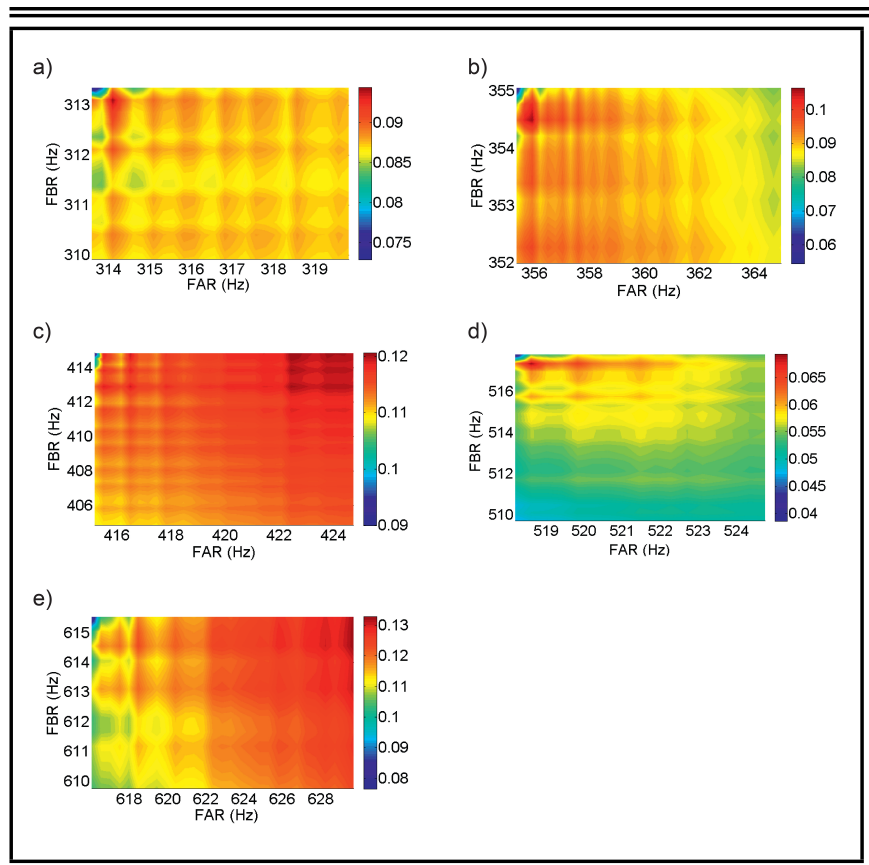

Figure 9. Evolution of loss factor for the first-five modal damping (a) $\xi=$ 0.0435 ; (b) $\xi=0.0448$; (c) $\xi=0.0575$; (d) $\xi=0.0275$; (e) $\xi=0.0533$.

between the impedance head and the tire. Responses at the surface of the tire were measured using accelerometers. There are three accelerometers used for the experiment; one is attached to the impedance head to measure the point mobility as shown in Fig. 10(b) while the other two are attached onto the tire with wax to measure the transfer mobility as shown in Fig. 10(c).

Figure 11 shows a comparison of the FRF between the FE simulation and the experimental results at both excitation and transfer points. At the excitation point, a wide variation in the natural frequency is noticed at the third mode between the FE simulation and the experimental result. The effect is also seen in the differences between their degrees of damping. At the transfer point, the peak height of the experimental result for each mode is slightly higher than the FE simulation.

In the experiment, the FRF contains both magnitude and phase information. The magnitude is typically shown on a logarithmic $\mathrm{Y}$ axis in decibels $(\mathrm{dB})$ scale, and the phase is often shown on a 0 to 360 degree scale. The phase often has shifts in degrees of discontinuities at each resonance frequency as illustrated in Fig. 12. The point inertance FRF was obtained through the spectral analyser. Figure 13 gives a close symmetrical relationship of the two transfer points used in the experiment. A small difference is observed because the positions of the two transfer accelerometers are not exactly symmetrical. Since every transfer point has to be measured, one could limit the measurements only to a half part of the tire and assume the same results for the other part. By this, a lesser time and cost to perform the experiment can be achieved.

The random excitation was also generated in the signal analyser. An advantage of random signal is that it removes nonlinear effects, noise, and distortion. Both the swept sine and the random give a similar reproduction of the behaviour of the inertance FRF at the three measured points. Figure 14 depicts the relationship between the swept sine inertance FRF and the random inertance FRF. It is also the case for the two transfer points.

In Table 4, a comparison is made between the modal analysis of the kart tire and the standard tires of their first five modes. For the kart tire, the results displayed are for an inflation pres-

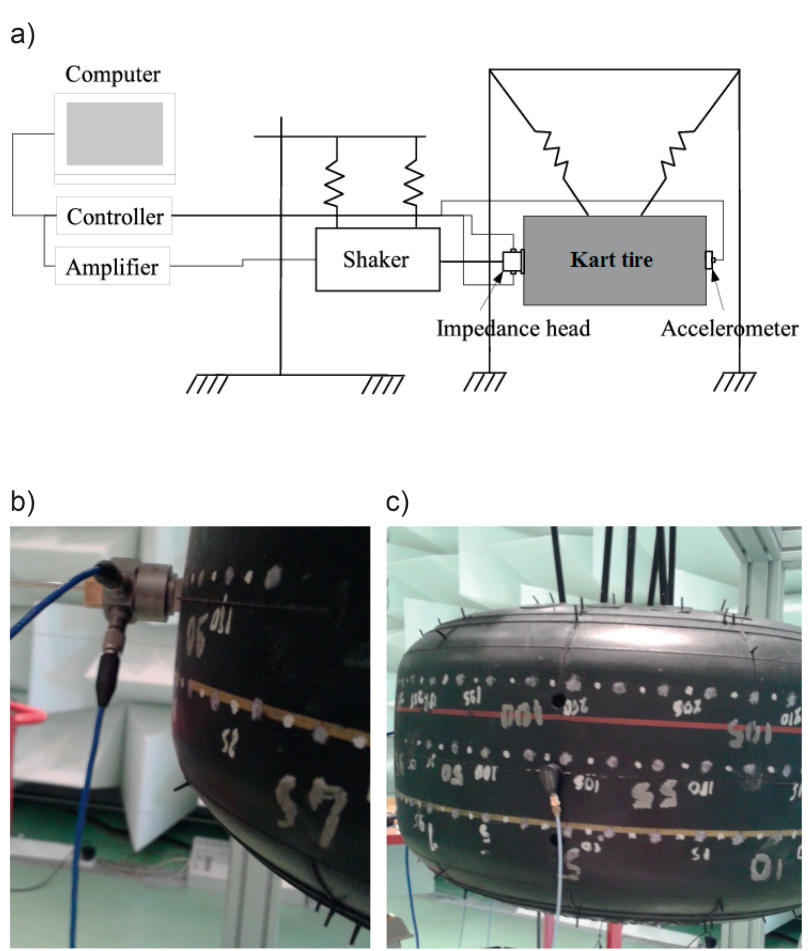

Figure 10. Experimental set up of suspended kart tire: (a) schematic diagram; (b) an accelerometer attached to the impedance head at the point of excitation; (c) two accelerometers attached at different transfer points.

sure of $200 \mathrm{kPa}$ at excitation point. The numerical result of Meftah for standard tires is for an inflation pressure of $250 \mathrm{kPa}$ cite21 while that of Tong and Jin is also for an inflation pressure of $250 \mathrm{kPa}^{22}$ Similarly, Pieters measured a standard automobile tire of dimension 205R $15 / 60$ at $160 \mathrm{kPa}$ and a static load of $4 \mathrm{kN} .^{12} \mathrm{In}$ his report, the range of frequencies obtained from mode 1 to mode 6 was between 122 to $276 \mathrm{~Hz}$. From these various result analyses, it is evident that the kart tire gives higher frequency range than the conventional ones. One of the possible reasons for the advantage of the kart tire over the standard tires is because of its simplified component parts. That is, only three components - tread, carcass, and steel beads are used for design and manufacturing of kart tires.

\section{CONCLUSION}

The finite element modal analysis introduced in this study provides an effective description in predicting the modal parameters of a small pneumatic kart tire. It shows the results of the mode shapes (Fig. 15), natural frequencies (Table 4) and damping ratios (Table 5) that emerge when a typical kart tire is excited under an applied force without contact to the ground surface and the motion of the tire rim is given a zero boundary condition. The mode shapes give the behaviour and responses of the tire during vibration. At first, the tire was given an excitation which caused it to vibrate until it reached its resonant frequency - also called natural frequency — for a small damping value. A transfer point was also located for the simulation and natural frequencies were obtained. The results of the finite element simulation show that for the kart tire, the frequency ranges from 300 to $850 \mathrm{~Hz}$ which is a considerably higher frequency range than that of the conventional standard tires, for which the frequencies are between 100 to $300 \mathrm{~Hz}$ during vibration with or without contact to the ground. Ex- 
Table 4. Natural frequency comparison between kart tire and standard tires.

\begin{tabular}{|c|c|c|c|c|c||}
\hline \multirow{2}{*}{ Mode } & \multicolumn{5}{|c|}{ Natural frequency (Hz) } \\
\cline { 2 - 6 } & \multicolumn{3}{|c|}{ Kart tire } & \multicolumn{3}{c||}{ Standard tires } \\
\cline { 2 - 6 } & Finite element & Experimental & Meftah $^{21}$ & Périsse et al. $^{20}$ & Tong and Jin \\
\hline 1 & 301.53 & 302.32 & 77.42 & 134.10 & 63.90 \\
\hline 2 & 361.08 & 360.48 & 100.66 & 158.70 & 90.30 \\
\hline 3 & 478.47 & 416.95 & 117.36 & 187.30 & 115.80 \\
\hline 4 & 517.97 & 512.67 & 134.63 & 220.10 & 141.80 \\
\hline 5 & 630.24 & 623.70 & 153.78 & 256.30 & 167.50 \\
\hline
\end{tabular}

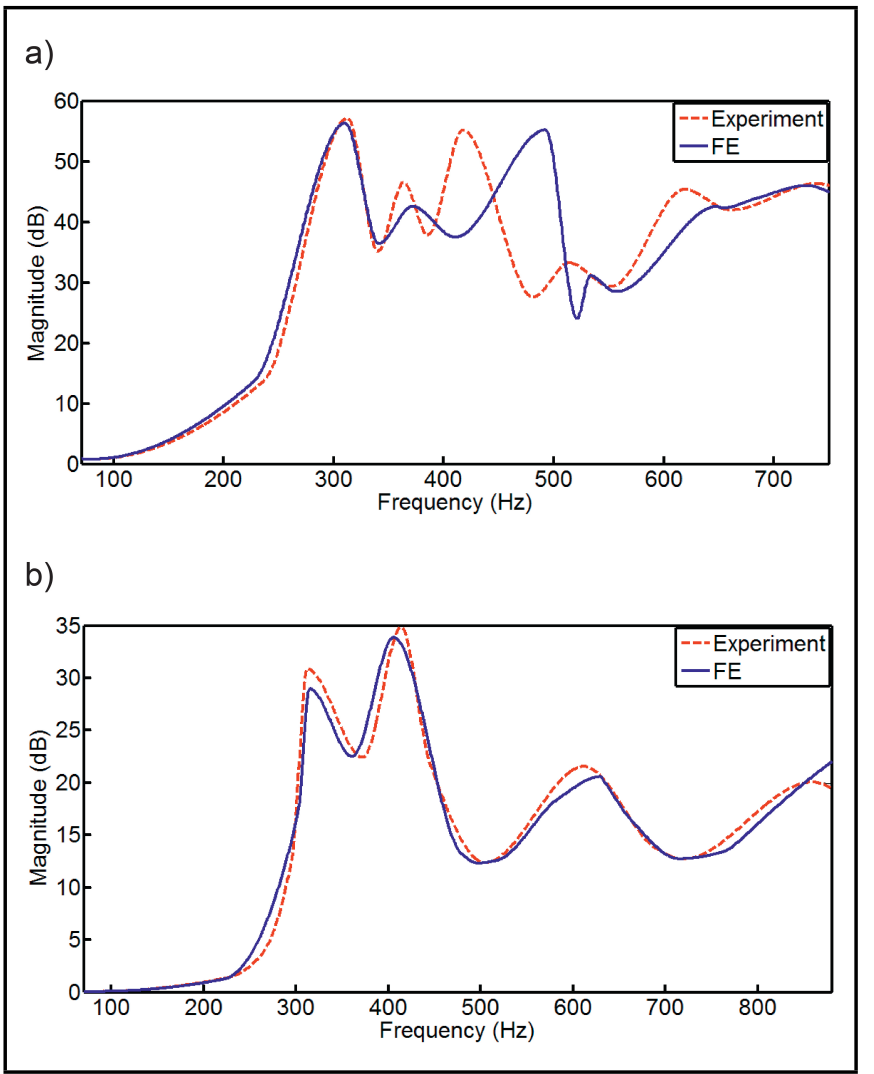

Figure 11. FRF comparison between experimental result and FE simulation: (a) excitation point and (b) transfer point.

Table 5. Damping ratio comparison between experimental results and FE simulation.

\begin{tabular}{|c|c|c|}
\hline \multirow{2}{*}{ Mode } & \multicolumn{2}{|c|}{ Damping ratio (\%) } \\
\cline { 2 - 3 } & Finite element & Experimental \\
\hline 1 & 4.18 & 4.35 \\
\hline 2 & 4.55 & 4.48 \\
\hline 3 & 6.63 & 5.75 \\
\hline 4 & 2.75 & 2.75 \\
\hline 5 & 5.46 & 5.33 \\
\hline
\end{tabular}

perimental results obtained from the FRF analyser validated the finite element solution. The higher natural frequencies obtained by this present work are good starting values that can be used for predicting tire-road contact, tire vibration, and airpumping. However, there is a limitation with the approach adopted in this study to effectively model rolling noise radiation which requires higher frequencies than those obtained in this present work.

\section{AKNOWLEDGEMENTS}

The financial support of the Institute Franais des Sciences et Technologies des Transports de l'Amangement et des Reseaux (IFSTTAR) Nantes; and the technical assistance by Dr. Cesbron Julien of the Laboratoire d'Acoustique Environnemen-

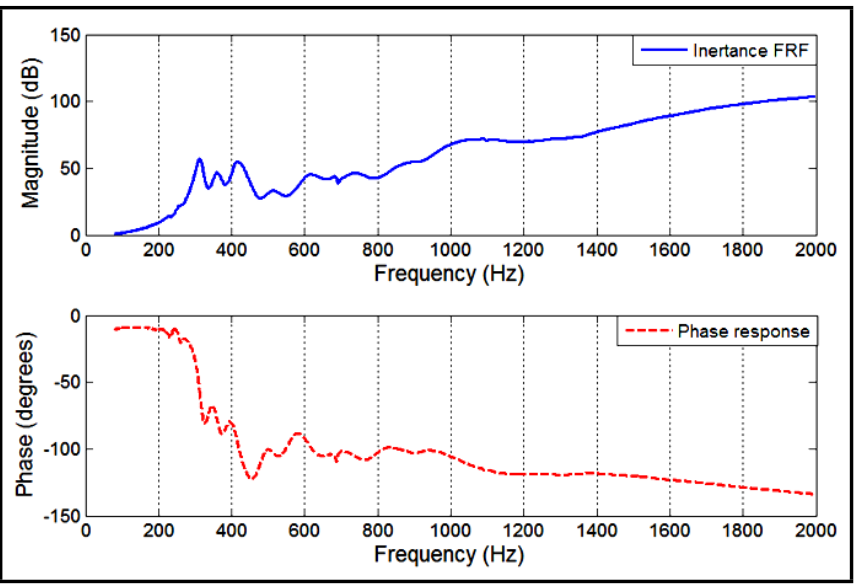

Figure 12. Excitation point inertance FRF showing the phase information.

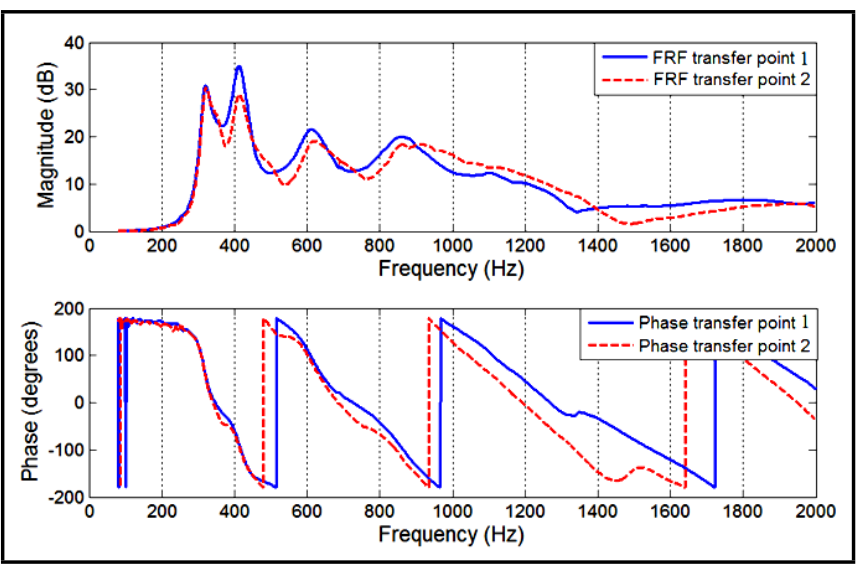

Figure 13. Transfer point inertance FRF showing the phase information.

tale, IFSTTAR Nantes, is gratefully acknowledged.

\section{REFERENCES}

1 Mace, B. R., Duhamel, D., Brennan, M. J., and L. Hinke. Finite element prediction of wave motion in structural waveguides, J. Acoust. Soc. Am., 117(5), 2835-2843, (2005). https://dx.doi.org/10.1121/1.1887126

2 Finnveden, S. Evaluation of modal density and group velocity by a finite element method, J. Sound. Vib., 273, 51-75, (2004). https://dx.doi.org/10.1016/j.jsv.2003.04.004

3 Chatterjee, A., and Ranjan, V. Free vibration analysis of radial pneumatic tire using FEM, Int. J. Emerg. Tech. Adv. Eng., 2 (8), 319-324, (2012).

4 Brinkmeier, M., Nackenhorst, U., Petersen, S., and Estorff, O. A nite element approach for the simulation of tire rolling noise, J. Sound. Vib., 309, 20-39, (2008). https://dx.doi.org/10.1016/j.jsv.2006.11.040 


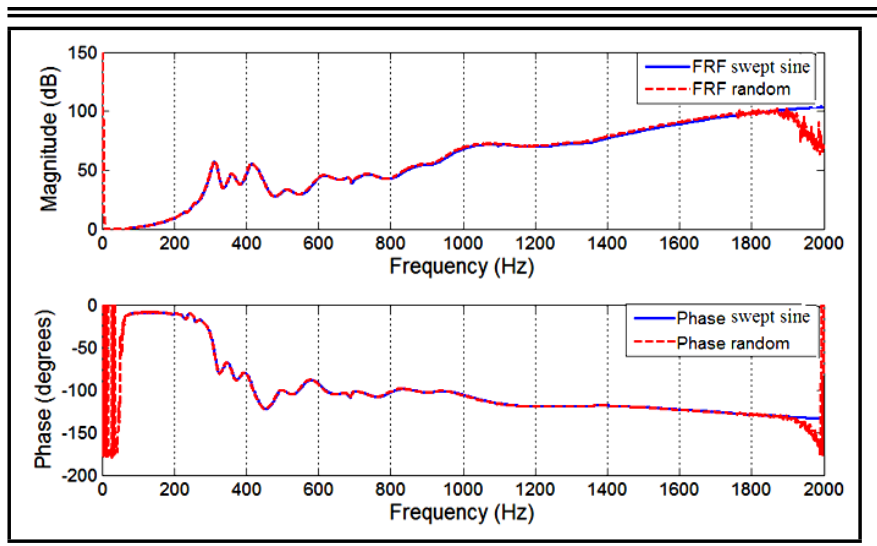

Figure 14. Comparison between the swept sine and random point inertance FRF.

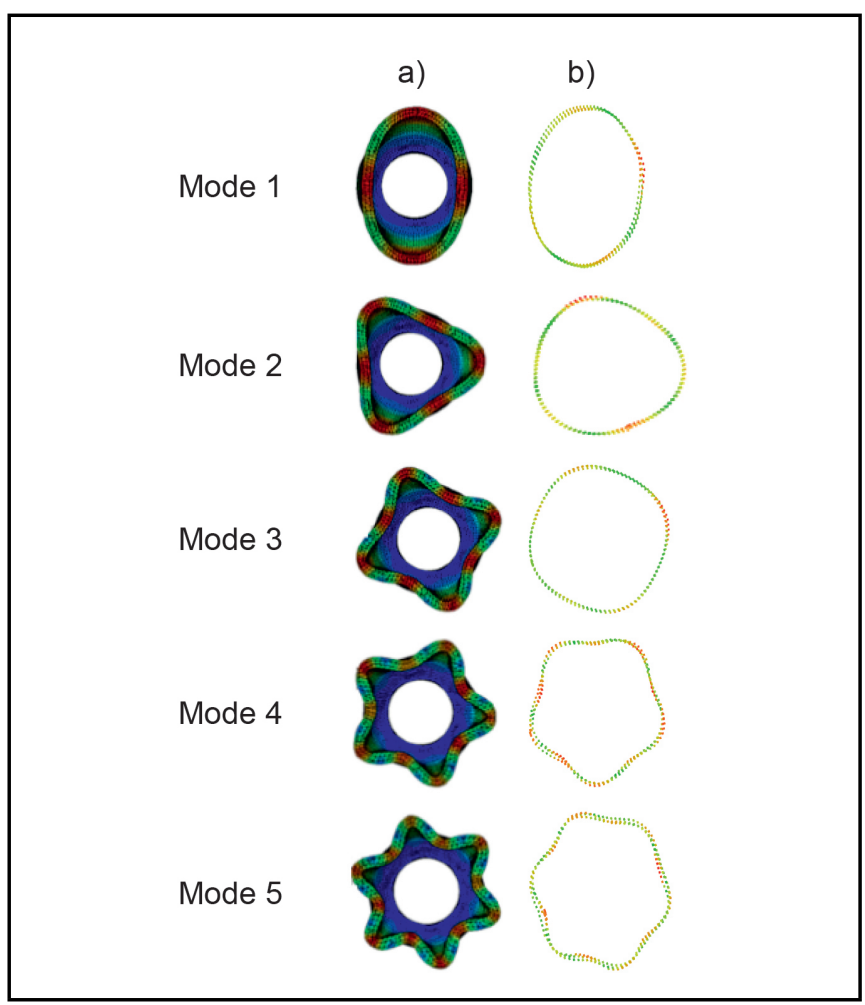

Figure 15. Modal shapes comparison between experimental result and FE simulation of kart tire for the first-five modes: (a) finite element tire modes section and (b) experimental tire modes.

5 Yoshiyuki, W., Brian, M., and Michael, B. Vibration analysis of a tyre model using the wave nite element method, International Congress on Acoustics, 19, 1-6, (2007).

6 Perisse, J., and Hamat, J. F. A comparison of the $2 \mathrm{~d}$ ring and $3 \mathrm{~d}$ orthotropic plate for modeling of radial tire vibrations, Internoise, France, (2000).

7 Lopez, I., Kersjes, S., Roozen, N. B., Schmeitz, A. J. C., and Nijmeijer, H. Green's functions for a rotating tire, $A$ semi-analytical approach, Euro Noise, Tampere, Finland, (2006).

8 Lopez, I., Blom, R.E.A., Roozen, N. B., and Nijmeijer, H. Modeling vibrations on deformed rolling tires a modal approach, J. Sound. Vib., 307, 481-494, (2007). https://dx.doi.org/10.1016/j.jsv.2007.05.056

9 Lopez, I., van Doorn, R. R. J., van der Steen, R., Roozen, N. B., and Nijmeijer, H.. Frequency loci veering due to de- formation in rotating tires, J. Sound. Vib., 324, 622-639, (2009). https://dx.doi.org/10.1016/j.jsv.2009.02.038

10 Kim, Y. J., and Bolton, J. S. Effects of rotation on the dynamics of a circular cylindrical shell with application to tire vibration, J. Sound. Vib., 275, 605-621, (2004). https://dx.doi.org/10.1016/j.jsv.2003.06.003

11 Koštial, P., Jančíková, Z., Bakošová, D., Valíček, J., Harničárová, M., and Špička, I. Artificial neural networks application in modal analysis of tires, Meas. Sci. Rev., 13 (5), 273-278, (2013). https://dx.doi.org/10.2478/msr-20130040

12 Pieters, R. S. Report No. DCT 2007.112, Experimental Modal Analysis of an Automobile Tire under Static Load, Eindhoven University of Technology, Department of Mechanical Engineering, Dynamics and Control Group, (2007).

13 Feng, Z., Gu, P., Chen, Y., and Li, Z. Modeling and experimental investigation of tire cavity noise generation mechanisms for a rolling Tire, SAE Int. J. Passeng. Cars Mech. Syst., 2(1), 1414-1423, (2009). https://dx.doi.org/10.4271/2009-01-2104

14 Time Domain Viscoelasticity, ABAQUS Analysis User's Manual Version, Dassault System.

15 Yang, X. Finite element analysis and experimental investigation of tyre characteristics for developing strain-based intelligent tyre system. $\mathrm{PhD}$ thesis, University of Birmingham, 2011.

16 Chae, S. Nonlinear finite element modeling and analysis of a truck tire. PhD thesis, The Pennsylvania State University, 2006.

17 Bilošová, A. Modal testing. Investment in Education Development, Ostrava, 2011.

18 Andersson, P. and Larsson, K. Validation of a high frequency three-dimensional tire model, Acta Acustica United with Acustica, 91, 121-131, (2005).

19 Soedel, W. and Prasad, M. G. Calculation of natural frequencies and modes of tires in road contact by utilizing eigenvalues of the axisymmetric noncontacting tire, J. Sound. Vib., 70, 573-584, (1980). https://dx.doi.org/10.1016/0022-460x(80)90325-9

20 Périsse, J., Clairet, J., and Hamet, J. Modal testing of a smooth tire in low and medium frequency - estimation on structural parameters, IMAC XVIII Conference, 2000.

21 Meftah, R. Une approach par formalisme de green rduit pour le calcul des structures en contact dynamique: application au contact pneumatique/chausse. PhD thesis, Universit Paris-Est, 2011.

22 Tong, G. and Jin, X. Study on the simulation of radial tire wear characteristics, WSEAS Transactions on Systems, 11 (8), 419-428, (2012).

23 Cosson, P. and Rozycki, P. Dynamique and Vibrations DYVIB,. Ecole Centrale de Nantes, 2013.

24 Ewins, D. J. Modal Testing: theory, practice and application. Research Studies Press LTD, 2nd edition, 2000. 